\title{
Improved method for complete gas-brine imbibition relative permeability curves
}

\author{
M. Ben Clennell ${ }^{1,}$, Cameron White ${ }^{1}$, Ausama Giwelli ${ }^{1}$, Matt Myers ${ }^{1}$, Lionel Esteban ${ }^{1}$, Michael Cullingford ${ }^{2}$, William \\ Richardson ${ }^{3}$, Gavin Ward ${ }^{2}$, Matt Waugh ${ }^{2}$, Scott Cole ${ }^{2}$, Ashley Hunt ${ }^{2}$ and Peter Bright ${ }^{2 \S}$
}

${ }^{1}$ CSIRO Energy, Australian Resources Research Centre, Kensington, Western Australia 6076

${ }^{2}$ Chevron Australia, QV1, 250 St George's Terrace Perth, Western Australia, 6000.

${ }^{3}$ Chevron Energy Technology Centre, 3901 Briarpark Drive, Houston, Texas 77042 USA

\begin{abstract}
Standard test methods for measuring imbibition gas-brine relative permeability on reservoir core samples often lead to non-uniform brine saturation. During co-current flow, the brine tends to bank up at the sample inlet and redistributes slowly, even with fractional flow of gas to brine of 400:1 or more. The first reliable Rel Perm point is often only attained after a brine saturation of around $\mathrm{S}_{\mathrm{w}}=40 \%$ is achieved, leaving a data gap between $S_{\text {wirr }}$ and this point. The consequent poor definition of the shape of the Rel Perm function can lead to uncertainty in the performance of gas reservoirs undergoing depletion drive with an encroaching aquifer or subjected to a water flood. We have developed new procedures to pre-condition brine saturation outside of the test rig and progress it in small increments to fill in the data gap at low $\mathrm{S}_{\mathrm{w}}$, before continuing with a co-current flood to the gas permeability end-point. The method was applied to series of sandstone samples from gas reservoirs from the NW Shelf of Australia, and a Berea standard. We found that the complete imbibition relative permeability curve is typically ' $\mathrm{S}$ ' shaped or has a rolling over, convex-up shape that is markedly different from the concave-up, Corey Rel Perm curve usually fitted to SCAL test data. This finding may have an economic upside if the reservoir produces gas at a high rate for longer than was originally predicted based on the old Rel Perm curves.
\end{abstract}

\section{Introduction}

When using standard, best-practice SCAL testing methods for measurement of imbibition gas-brine relative permeability on reservoir core samples, e.g., as recommended by McPhee et al. [1], it is very difficult to establish a uniformly progressing brine saturation. This difficulty in establishing stable co-current flow conditions leads to poor definition of the shape of the Rel Perm function at lower brine saturations when gas has the highest mobility.

Typically, during co-current flow with forced imbibition of brine along with gas, the saturation within the sample does not advance uniformly along the sample length but will bank up at the inlet end of the sample (Figure 1). The brine largely builds up by spontaneous imbibition from the restricted supply available and owing to low $k_{\mathrm{rw}}$ it takes hours or days for brine to redistribute along the sample length, even with high fractional flow of gas to brine of 400:1 or more. Moreover, the first reliable co-current gas-brine relative permeability point (stable differential pressure and uniform saturation) is often only attained after a brine saturation of $\mathrm{S}_{\mathrm{w}}=40 \%$ or more is achieved, leaving a significant data gap between $S_{w i r r}$ and this point (Figure 2).

The data in Figure 2 can be interpolated with a concave-up Corey function curve [2], but this may be incorrect. The lack of valid data can lead to questionable inputs to (and outputs of) reservoir simulation models and flow on to uncertainty in the performance of gas reservoirs undergoing depletion drive with an encroach-

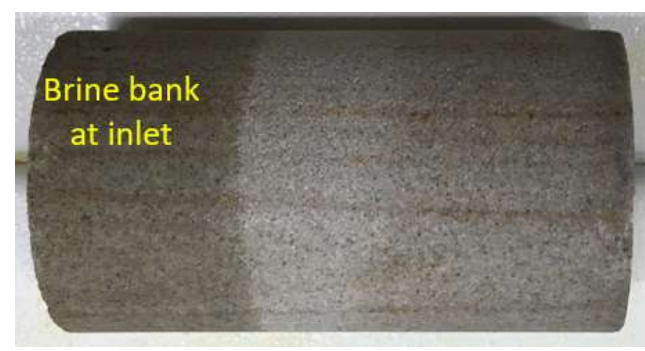

Fig. 1. Berea sandstone sample taken from test rig at the early stage of an imbibition Rel Perm measurement using standard, co-current SCAL methods. A large brine bank has formed at the inlet that blocks the permeability to gas.

\footnotetext{
* Corresponding author: Ben.Clennell@ csiro.au $\quad$ SPresent Address: BHP Petroleum, 125 St George's Terrace, Perth W.A. 6000.
} 


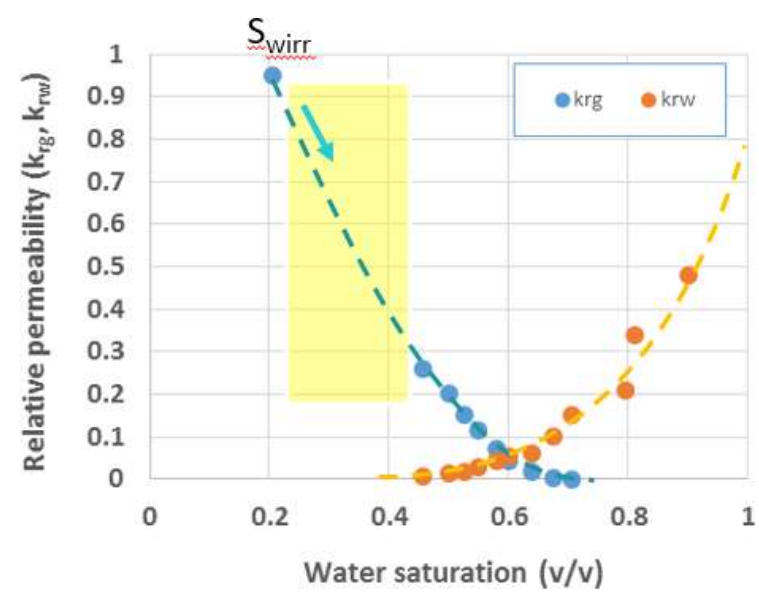

Fig. 2. Example of a good quality, but incomplete imbibition relative permeability curve. There is a large data gap (yellow) in the gas Rel Perm data between the gas-only flow point at $S_{\text {wirr }}$ and the first co-current flow point at $S_{\mathrm{w}}=0.43$. Data from [3].

-ing aquifer or subjected to a water flood.

The general problem of incomplete imbibition Rel Perm curves available for conventional gas field development planning has been pointed out previously at this forum [2] but it has not so far been resolved in the form of a more practical measurement method that can obtain accurate and complete Rel Perm curves at low to intermediate $S_{\mathrm{w}}$.

In 2016 CSIRO was engaged by industry partners to investigate improvements to Special Core Analysis (SCAL) test methods for the purpose of reducing uncertainties in the development of major gas fields in the Northwest Shelf of Australia. During the ensuing research project, CSIRO worked with the operating company and in-house domain experts to devise and test new laboratory methods. We were able to collect a series of much more complete imbibition gas-brine relative permeability curves on several sandstone samples of moderate to excellent quality. The methods were applied to five NWS gas reservoir sandstones and also to a Berea sandstone, a well characterized "standard" rock, which produced similar results.

\section{Materials and Methods}

Experiments were conducted on reservoir sandstone samples ranging in dry gas permeability from $10 \mathrm{mD}$ to around $670 \mathrm{mD}$, and with porosity ranging from $17 \%$ to $23 \%$ (see Figure A1). The reservoir sandstones are all quartz arenites $(\mathrm{Q}>78 \%$ ) with 6-10\% feldspar and 3-4\% kaolinite as the only clay mineral. The Berea sandstone outcrop sample used was quartz rich $(\mathrm{Q}>90 \%)$ and well sorted with dry gas permeability of $665 \mathrm{mD}$ and porosity of $21.8 \%$. Core plugs of around 50-60 mm length and 38 $\mathrm{mm}$ in diameter were cleaned prior to measurement, and then saturated with synthetic formation brine.

The samples were drained to irreducible brine saturation using a petrophysical centrifuge up to either 100 psi or 250 psi air-brine capillary pressure. The imbibition Rel Perm experiments were commenced from close to the value of irreducible brine saturation $S_{\text {wirr }}$, which in the case of the Berea sample and the most permeable reservoir sample, was around $2 \%$, for the other reservoir samples $S_{\text {wirr }}$ ranged from 10 to $15 \%$.

Samples were loaded into the CSIRO x-ray CT monitored core flooding rig (Figure 3). The system uses accurate differential (Keller, Switzerland) and absolute (Quartzdyne, USA) pressure transducers capable of resolving a very small pressure drop across the sample, and gas (Teledyne ISCO, USA) and brine (Quizix, Chandler USA) pumps with high accuracy and a wide range of allowable flow rates $(0.001$ to $120 \mathrm{cc} / \mathrm{min})$. Flow into and out of the sample is by means of two porous end stones that are removable for cleaning and for controlling their initial brine content. The pressure drop for flow calculations is across the sample only, with the end stones bypassed by thin peek tubes and attached to the differential pressure transducer (Figure 4).

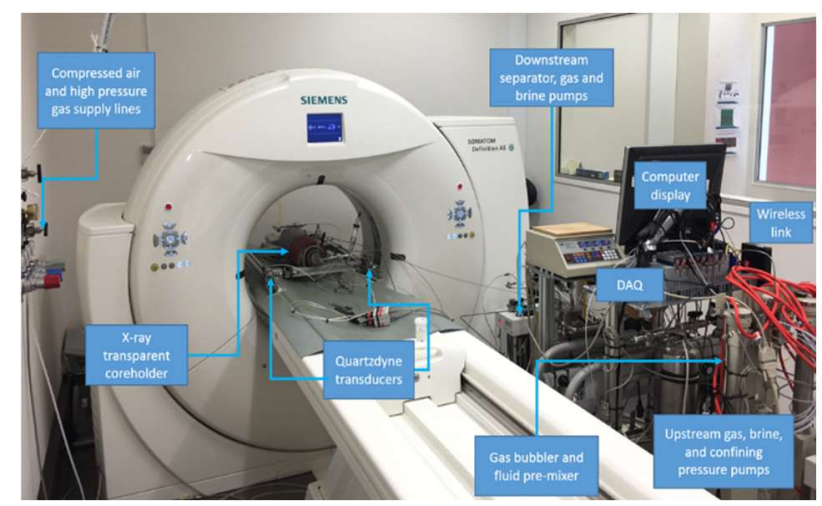

Fig. 3. CSIRO x-ray CT monitored core flooding rig.

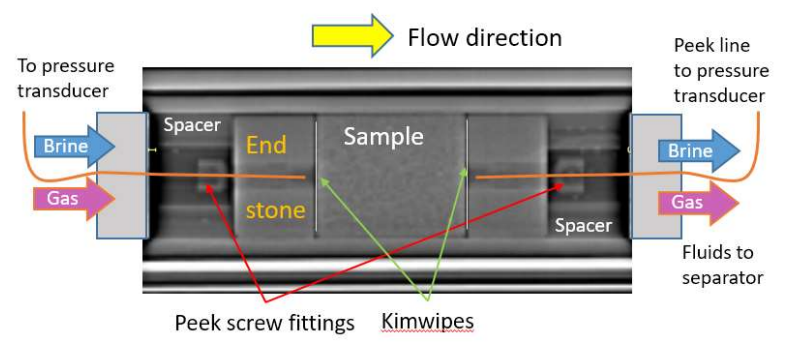

Fig. 4. Configuration of the core holder with removeable end stones made of Bentheimer sandstones that served as flow distributors for gas and brine. Pressure drop is measured only across the sample itself via a thin PEEK tube. Single ply tissue was used for capillary continuity at the ends of the sample.

In all the tests reported here a confining pressure of $2500 \mathrm{psi}$ and a pore pressure of $500 \mathrm{psi}$ were used. The gas used was nitrogen rather than methane for safety reasons. A sodium iodide-based brine was used to enhance $\mathrm{x}$-ray contrast, though the overall composition was tested to be compatible with any clays in the rocks. The saturation computations from x-ray CT numbers used calibrations from dry and brine-saturation samples loaded to the same net overburden pressure of 2000 psi used in the test. Moreover, we also used intermediate points of known saturation before applying back pressure, to better constrain the calibration. The saturation from XCT is determined to within $3 \%$, with errors from inexact repositioning of the sample, and drift in beam energy. 
When using the standard technique of high gas fraction/ low brine fraction co-current flow to introduce the water in sample S22B, we encountered the same problem that has been reported previously. The brine entering the rock accumulated at the inlet and did not distribute uniformly until $S_{w}$ of over $50 \%$ had been achieved. By this stage, the gas was nearly immobile. The relative permeability is poorly defined (Figures $5 \& 6$ ).

In these figures the valid Rel Perm data points are shown in solid colour. The data point at $S_{w}=49 \%$ was collected when pressure was stable and the flow rates into

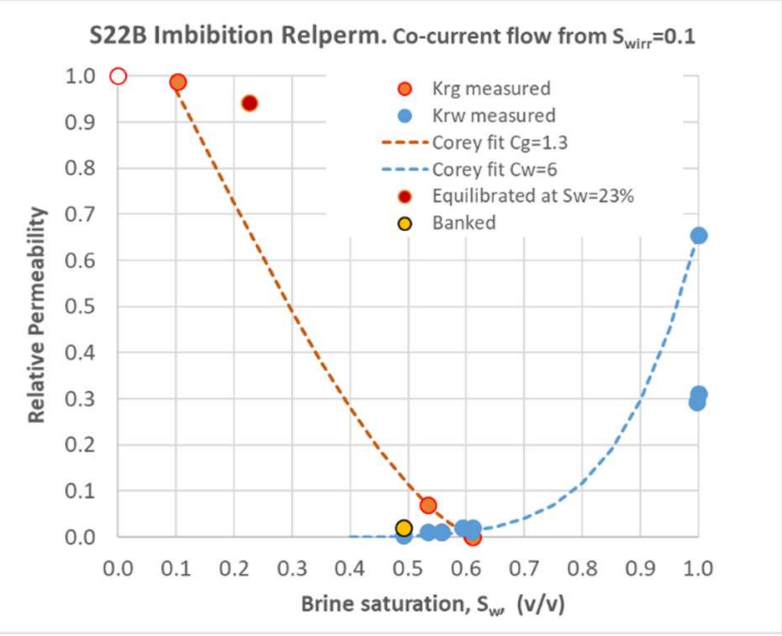

Fig. 5. Relative permeability curve for reservoir sandstone of good quality, (dry gas permeability $107 \mathrm{mD}$ ), collected using standard co-current test method. A Corey function with small exponent $C_{\mathrm{g}}=1.3$ can be used to fit the valid data points but there is a large gap between $k_{\mathrm{rg}}$ at $\mathrm{S}_{\text {wirr }}$, around $103 \mathrm{mD}$, and the next point where the brine was seen on the x-ray CT to be evenly distributed along the length of the sample.

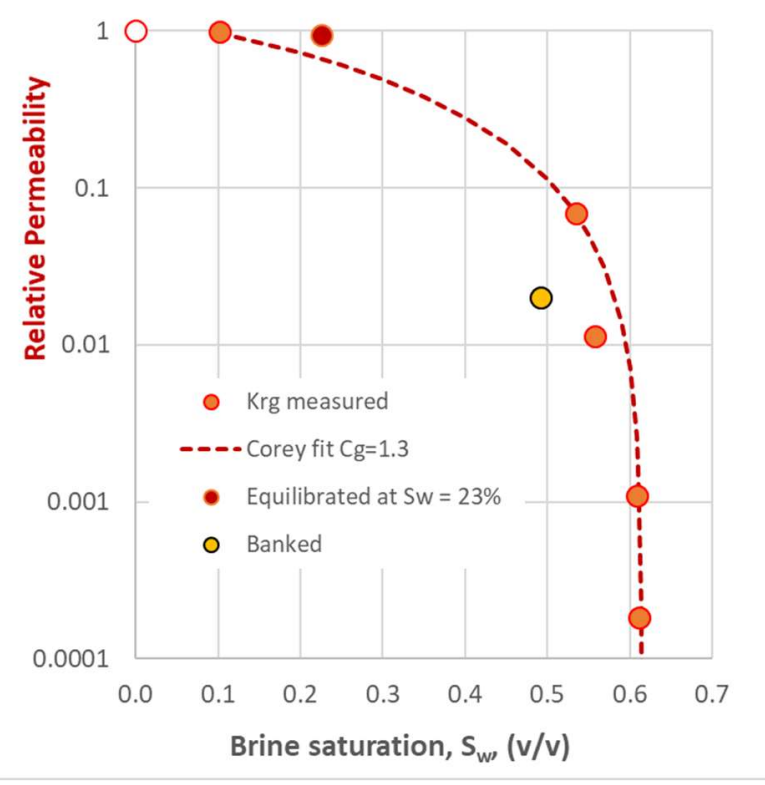

Fig. 6. Gas-only Rel Perm data in Figure 5, plotted in an expanded logarithmic scale. Points at $\mathrm{S}_{\mathrm{w}}=0.23$ (imbibed) and 0.49 (banked) are discussed in the text. and out of the sample were equal. However, the x-ray images (Figures A7-A9) show that the saturation profile was not even at that time. A brine bank formed at the inlet had not yet dissipated (Figure A7 image 2). The high brine saturation blocked gas flow through the sample, so that its relative permeability was only $2.2 \mathrm{mD}$. This banked point is labelled with a black border. During continued cocurrent flow at 400:1 gas to brine ratio the bank spread out through the sample (in fact there was locally, some secondary drainage of excess brine) and a valid point was eventually achieved at $\mathrm{S}_{\mathrm{w}}=53 \%$, with $k_{\mathrm{rg}}=7.6 \mathrm{mD}$.

To overcome the problems described above, we developed simple procedures to create a more uniform brine saturation in the low $S_{w}$ region, but otherwise retain the desirable features of steady-state Rel Perm procedures established as best practice and outlined, e.g. in McPhee et al. [1]. The new step is to control the early imbibition process in small increments of $5-10 \% \mathrm{~S}_{\mathrm{w}}$ outside of the test cell. We achieved this by blotting a known amount of brine slowly into the rock plug from all sides with lintfree laboratory tissue (green Kimwipe ${ }^{\mathrm{TM}}$ ), wrapping the sample in the tissue followed by a layer of polyethylene cling film and allowing it to imbibe and redistribute evenly. This took several hours for permeable samples) while several days was found necessary for the least permeable samples. Several samples were treated in parallel so that they could be loaded sequentially into the test rig and then each advanced further. This means that test rig throughput remains high.

The use of removeable end stones made from highly uniform Bentheimer sandstone meant that the brine saturation in the sample could be pre-equilibrated with the flow boundary materials, also outside of the rig, for each particular sample. We used hand-tightened ' $G$ ' clamps to press the end stones against the test samples, while imbibition of the inlet-sample-outlet composite took place. We used mouldable wax film (Parafilm $^{\mathrm{TM}}$ ) wrapped around the polyethyene cling film to keep the blotting tissue firmly in place around the samples and stones, and the whole assembly was stored horizontally in the refrigerator.

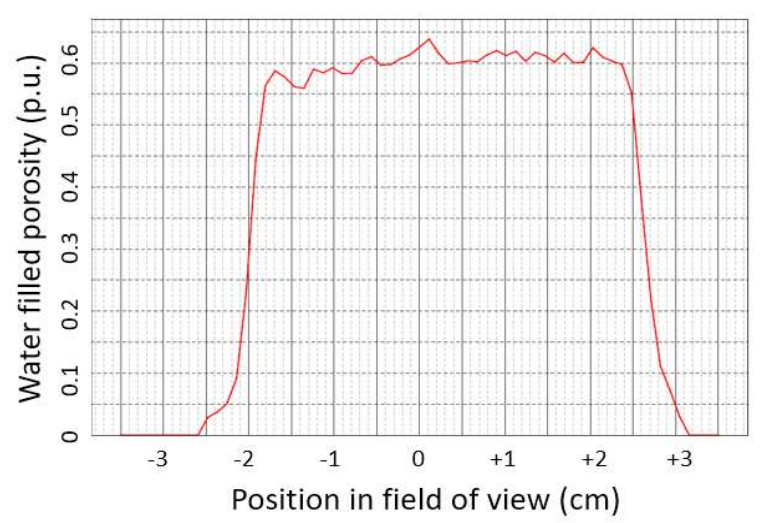

Fig. 7. Example of NMR saturation profile through core plug of high permeability sandstone after blotting with tissue and equilibration. Tissue was removed prior to NMR scan acquisition. Good uniformity in water filled porosity along the core length shows that an even saturation of brine through the sample has been achieved. 
Uniform brine saturation throughout the rock was verified using NMR $T_{2}$ spectroscopy and profiling along the axis of the cling film-wrapped sample (Figure 7).

The equilibrated samples were loaded into the x-ray imaging flow rig and confining pressure and back pressure were applied slowly. Figure A2 shows an example of a high permeability sandstone together with its end stones loaded into in the test rig.

With the blotting procedure, a series of data points was collected to fill in the data points from the starting point, at or close to $S_{\text {wirr }}$, up to intermediate brine saturations.

At low $S_{w}$, before brine became substantially mobile, we injected gas-only, at four or more rates, to define accurate gas relative permeability values that we also found to be highly repeatable. That is, we avoided cocurrent flow to avoid banking and we used humidified gas to prevent drying of the samples. Using multiple rates, the linearity of the flow (obeying Darcy's Law) could be confirmed, and any offsets in the transducer values (instrument drift and capillary effects not indicative of the flow-induced pressure drop; e.g. see [5], [6]) could be tracked. Generally, we found that capillary effects close to the inlet or outlet upset the stability of the pressure records to such an extent that the data was unusable (Figure A3). Therefore, we developed a procedure to bleed out any brine droplets that were trapped in the inlet of the PEEK tubes and were able to collect very stable pressure readings resolving pressure drops smaller than 0.02 psi. Figure A4 shows an example of a good quality record in a high permeability sample where very small differential pressure signals can be resolved.

Once saturation had advanced sufficiently for brine to be detectably mobile, we progressed into co-current flows with the fractions of gas and brine adjusted to maintain near stable, but slowly advancing $\mathrm{S}_{\mathrm{w}}$. We note that commonly, onset of brine mobility coincided with nonlinearity of the pressure response to increased flow rate: in these cases, we used the linear portion of the curve at lower gas flow rates to compute the relative permeability.

The use of paired syringe pumps at inlet and outlet allowed close control of both inflow and outflow brine rates, such that true steady state conditions were verified. We define a point as "valid" when flow rates in and out of the sample are equal, the differential pressure is steady, and the saturation is uniform to within five percent. Points that are invalid or suspect are shaded grey on the plots.

Ideally, the brine saturation should be completely uniform and, in many cases, $\mathrm{S}_{\mathrm{w}}$ did vary by less than $2 \%$ along the sample length. We found that in the highly permeable sandstones, the brine was easily moved within the sample during loading and pressurization in the rig. As $\mathrm{S}_{\mathrm{w}}$ was increased beyond about $50 \%$, brine became more mobile, and there was a tendency to see some end effects so that brine either accumulated at, or retracted from, the sample boundaries: if this led to a saturation range greater than $5 \%$ within the sample we consider it invalid. Note that "invalid" points happen to lie on the curves eventually fitted through the valid data points.

The x-ray images of the experiments taken in the medical CT system included the end stones and this gave us advanced warning of potential saturation changes that could be balanced with a higher or lower brine flow rate.
In this way, the progression of saturation was made as smooth as possible and the imbibition was controlled to be monotonic (meaning without any regressions in water saturation owing to too-rapid drainage of brine out of the sample). Even so, the experiments are quite challenging to perform as uneven brine saturation can still occur due to brine accumulating at the inlet (banking, as described above, owing to spontaneous imbibition from the upstream stone) or the outlet end of the sample (likely due to poor capillary continuity between the sample and the downstream stone).

\section{Results}

We present representative results from our study for three samples representing low, intermediate and good quality gas reservoir types.

In Figures 5 and 6 we illustrate the effect of resetting the saturation of Sample S22B from $\mathrm{S}_{\text {wirr }}$ and advancing

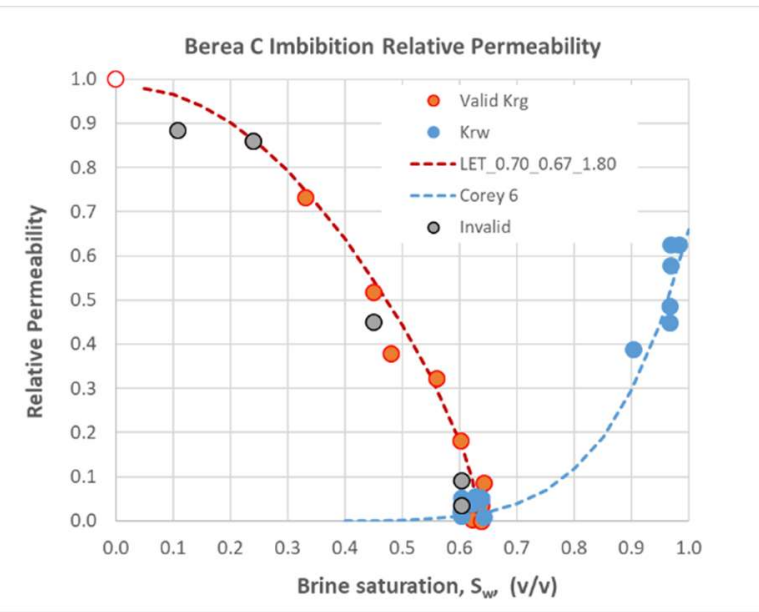

(a)

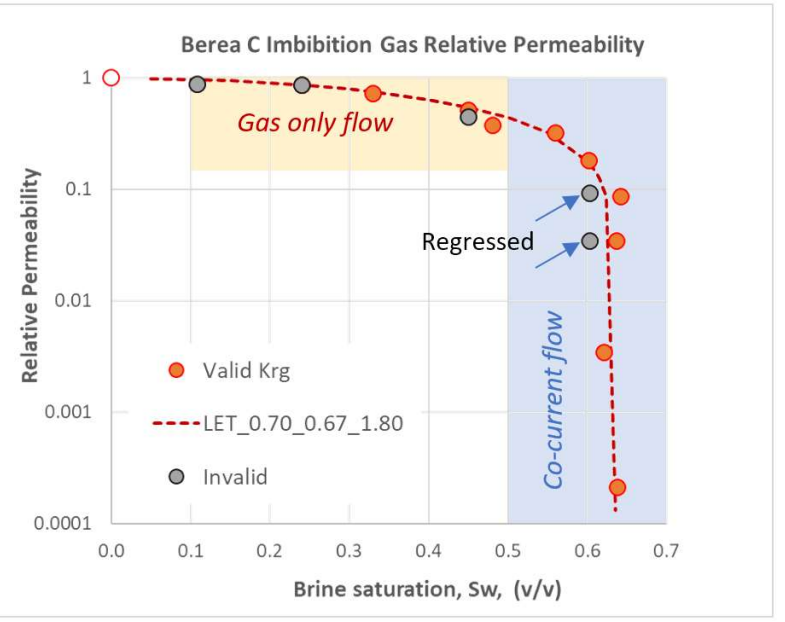

(b)

Fig. 8. Gas relative permeability (orange dots are valid points) and brine relative permeability (blue dots) for Berea $\mathrm{C}$ sample. The red dashed line is the LET function fit to gas permeability. Brine data in (a) fit a Corey function. In the expanded, logarithmic-scale plot (b), the location of two stable and equilibrated points that lie below the main Rel Perm curve are labelled. They were acquired after a period of regression in the brine saturation during co-current flow. 
$\mathrm{S}_{\mathrm{w}}$ to $\sim 23 \%$ using the blotting method to collect just one additional equilibrated imbibition Rel Perm point within the gap region. This point is shown in darker solid colour, and it lies well above the Corey curve fitted through the other points acquired earlier with the standard methodology. The Corey fit was allowable when there was a large data gap, but the new point makes this type of curve impossible. By collecting more points with sequential blotting, we can trace a more complete curve towards $\mathrm{S}_{\mathrm{w}}$ values of around $50 \%$ brine when the cocurrent data are again reliable.

Figure 8 shows the full results for Berea sandstone, which has a dry gas permeability of around $665 \mathrm{mD}$. The evolution of brine distribution for this sample is shown in Figure A5. This sample was challenging owing to high water mobility, which was near the limit of causing gravitational segregation during horizontal core floods. Two points at around 11 and $24 \%$ brine saturation showed more brine in the middle part of the core than at the ends, and therefore are coloured grey in Figure 8.

These two values were collected immediately after loading the samples into the rig, from an original state that did have uniform brine distribution, in equilibrium with the end stones. We presume that application of the gas pressure from both ends of the sample caused some retraction of the brine towards the middle of the core plug.

Figure 9 shows the gas relative permeability curve for the poorest quality reservoir sample, S26B (porosity $17 \%$, dry gas permeability of $9.4 \mathrm{mD}$ ). The first point was collected at $\mathrm{S}_{\mathrm{w}}=25 \%$, following centrifugation to $100 \mathrm{psi}$. The general shape of the Rel Perm curve is similar to the Berea and S22B but with slower fall-off and a gas end point at higher water saturation $\left(\mathrm{S}_{\mathrm{w}}=73 \%, \mathrm{~S}_{\mathrm{gr}}=27 \%\right)$.

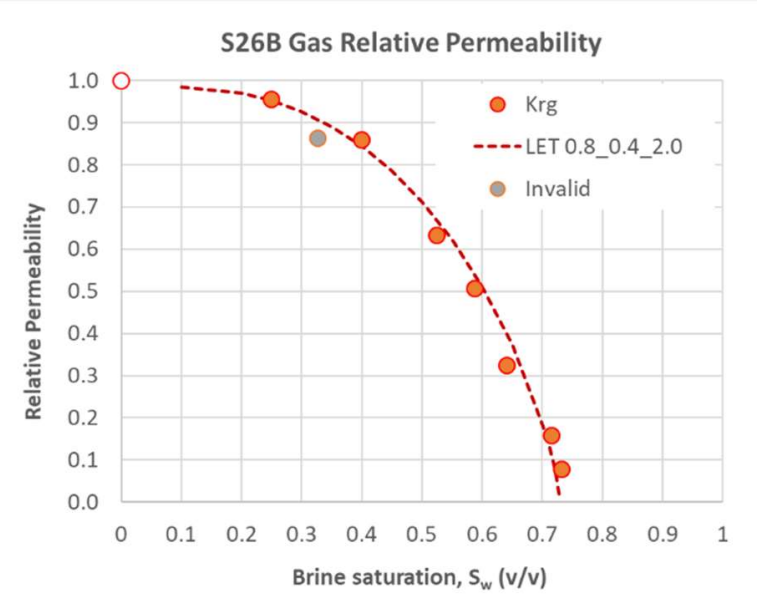

Fig. 9. Gas relative permeability (orange dots are valid points) for sample S26B. The red dashed line is the LET function fit.

For five reservoir sandstones and for the Berea "standard" rock we found that the shape of the more complete gas relative permeability curve, now extending through to low $S_{w}$ values, is convex upwards and does not show the concave-up or "Corey" type profile that is conventionally expected. The gas Rel Perm function may have an ' $S$ ' shaped form or simply roll over to a sharp drop-off without tapering at high $\mathrm{S}_{\mathrm{w}}$. The experimental gas Rel Perm curves can be fitted with the LET function [7], while the water Rel Perm curves still show a classic Corey function shape, as expected from established theory for strongly water wet conditions. The properties and LET fitting values for three representative samples are summarised in Table 1.

We note that during the co-current test periods, we saw several of the phenomena mentioned by Cense et al. [2] for gas-brine imbibition experiments, such as the development of a gradient in brine saturation along the sample near the permeability end point owing to greater compression of trapped gas bubbles at the inlet end than at the outlet end, and also a small amount of "carry-over" of gas in solution or as disconnected bubbles. We therefore used recommended procedures not allowing the differential pressure to exceed 50 psi $(10 \%$ of the back pressure applied). Usually $\Delta \mathrm{P}$ was less than 5 psi.

When regressions in saturation occurred, the gas relative permeability for the subsequent point lay below the curve for progressive imbibition, suggesting that additional blocking of gas pathways by displaced brine was occurring. The "invalid" grey points labelled with arrows between $S_{w}=0.4$ and $S_{w}=0.64$ in Figure $8 b$ were acquired after such episodes of regression in saturation during the co-current flow period in the Berea $\mathrm{C}$ sample.

\section{Discussion and Implications}

Our findings question the adequacy of current "best practice" relative permeability testing methods and reporting in SCAL programs for gas reservoirs. While every Rel Perm point reported may be valid, the limited data available are often fitted through the "gap" at intermediate $S_{w}$ values using a Corey-type curve that may not be applicable. (Figures A7 to A9 show how this gap came about in sample S22B using co-current flow at 1000:1 gas to brine ratio). Therefore, completing the curve in this region can reduce the uncertainty in what the permeability trajectory during brine imbibition will be.

The results that we obtained for a range of sandstones from good and poor-quality reservoirs suggest that the shape of gas-brine imbibition relative permeability curves should not be assumed to have a concave-up shape, at least for water wet systems such as those investigated in this research project. Considering that in a water wet rock, the brine has a strong affinity for surfaces, it is not surprising to us that the brine does not start to have a marked effect on the gas permeability until it has built up to a level where it blocks the gas pathways in the centres of pores and throat regions. Once the pathways start to block up however, the permeability to gas drops more precipitously in a process akin to the removal of conducting bonds in a percolating network.

Sigmoidal-shaped [imbibition] relative permeability curves for oil-brine and gas-brine system have been reported in the literature, e.g. [8-12], hence the derivation of fitting functions such as LET [7] to describe them. The shape is likely to relate to the pore structure and the "rollover" shape that has been termed "reverse hysteresis" is sometimes cited as being typical of a sand-pack rather than a heavily consolidated rock [13]. In simple network 
simulations of two-phase displacements, the shape is typical of low aspect ratio pore structures, and wellconnected topology $[14,15]$. We note that the porosity of the samples that we tested is from $\sim 17 \%-22 \%$, and microscopic examination shows moderate consolidation and quartz cementation, but in general that sandstones all have an "open" and well-connected type of pore structure.

The Berea sandstone sample that we tested here, is homogeneous in the along-core direction, and looks uniform on visual inspection but has discernible lamination in the x-ray images (Figure A6), whose contrast increases at intermediate brine saturations when the finer layers imbibe more brine relative to the coarse layers. Thus, for Berea $\mathrm{C}$, there are likely to be some relatively unrestricted channels for gas flow at intermediate brine saturation, that remain free flowing until those laminae with large pore apertures also become invaded by brine. This would act to maintain high gas of Rel Perm to a larger value of $S_{w}$ and generate a "roll over" curve shape thereafter. However, we should point out that all the other samples were chosen for their homogeneity and the overall shape of the permeability decline in laminated Berea $\mathrm{C}$ is rather similar to that measured for the highly uniform sample S26B.

Other factors such as interfacial tension, wettability and flow rates affecting the viscous force balance are likely to be as important as the pore structure in controlling the Rel Perm curve shapes. Kjosavic et al. [16] suggest that strong water wetting can produce a sigmoidal shape of Rel Perm curves and leads to an increase in hysteresis between drainage and imbibition processes. A decrease in the exponent describing the non-wetting phase tortuosity (which depends on pore structure, IFT and wettability) is shown by Gerhard and Keuper [13] to generate a convex-up shape for the first imbibition curve and for imbibition scanning curves: physically this implies that the gas flow pathways are relatively simple and dominant with the water phase forced to the margins of the pore space. More sophisticated measures of nonwetting phase continuity and connectedness now being made possible with $\mathrm{x}$-ray microCT image analysis at pore scale may help to put these measures on a more quantitative and predictive footing [17]. Indeed, digital rock simulations with realistic physics can already predict a range of Rel Perm curve shapes, and they can produce output continuously across the full range of saturations $[18,19]$.

Our results apply to the water wet conditions tested in the gas-brine system having high interfacial tension (around $70 \mathrm{mNm}^{-1}$ ) in quartzo-feldspathic sandstones with a small proportion of clay, and moderate to high permeability. The prevailing conditions of our tests are representative of the interior of the reservoir (far from a well) and involve small capillary numbers (ratio of viscous (drag) forces to capillary (surface) forces of around $10^{-6}$ or $10^{-7}$. At higher velocity, and capillary number $>10^{-5}$, the viscous forces and momentum transfer will change the shape of the gas Rel Perm curve, which is likely to straighten as the gas can more effectively push brine out of the way. Therefore, prediction of gas flow dynamics close to the wellbore would require a different style of lab test and would most likely lead to differently shaped Rel Perm curves than the examples reported here.

Considering the true sigmoidal or roll-over profile of the gas Rel Perm curves at low capillary number, one can see that the gas flow rates predicted for the earlier stages of brine invasion into the reservoir (away from the near wellbore region) as it is depleted or swept, will be higher than would be predicted by defaulting to the concave-up "Corey" shape. (Figure 5 shows an example a good Corey curve fit to limited data). The Corey and LET type curves only merge towards the tail end of gas mobility, when the relative permeability is less than 10 percent of the starting values at $S_{\text {wirr }}$. Therefore, one would predict that higher gas flow rates are possible in the reservoir during the early stages of water ingress, before a steeper drop-off in $k_{\mathrm{rg}}$. The consequences for reservoir planning are likely to be more important for a water drive situation and be less important for reservoirs only undergoing depletion.

This new understanding of the true gas-brine relative permeability curve shapes may influence the development concept for gas reservoirs, the placement of wells and even the ultimate economics of a project. At the least, having complete and reliable relative permeability curves as inputs will improve the accuracy of reservoir simulation models and increase the level of confidence in their predictions of reservoir performance over time.

\section{Acknowledgements}

Chevron Australia and joint venture partners Exxon Mobil Australia and Shell Australia are thanked for funding this research. Dr Ali Saeedi from Curtin University provided important guidance in setting up this project, and advice as to experimental methods. Dr Lincoln Paterson and Dr Tara La Force from CSIRO are thanked for providing internal peer reviews. P. Egermann and K. Flynn are thanked for thoughtful reviews that improved the quality of the paper. This research utilized the X-ray CT and Advanced Core flooding facilities of the National Geosequestration Laboratory (ngl.org.au).

\section{References}

1. C. McPhee, J. Reed and I. Zubizarreta, Core Analysis: A Best Practice Guide. Elsevier (2015).

2. A. Cense, J. Reed and P. Egermann. "SCAL for gas reservoirs: a contribution for better experiments". SCA 2016-023 (2016).

3. D. Maloney, K. Doggett and R. Gilliland. "New procedure and apparatus for combined reservoir condition gas-brine relative permeability, capillary pressure and electrical property measurements". SCA-9427 (1994).

4. A. T. Corey. "The interrelation between gas and oil relative permeabilities". Producers Monthly, 19, 3841, (1954).

5. R. Pini, and S. Benson. "Simultaneous measurement of capillary pressure and relative permeability drainage curves". Water Resources Research, 49, 3516-3530, (2013). 
6. R. Gupta, and D. Maloney. "Intercept Method-A novel technique to correct steady-state relative permeability data for capillary end effects". SPE171797-PA (2014)

7. F. Lomeland, E. Ebeltoft, and W.H. Thomas, "A new versatile relative permeability correlation". SCA 2005-32. (2005).

8. J. Osoba, J. Richardson, J. Kerver, J. Hafford, and P. Blair. "Laboratory Measurements of Relative Permeability". Petroleum Transactions AIME, 192, 47-56 (1951).

9. J. Batycky and F. McCaffery. "Low interfacial tension displacement studies". Petroleum Society of CIM, Calgary, Canada, Paper 78.29.26. 1978.

10. Raimondi, P. "Distribution of the oil phase obtained upon imbibition of water". Society of Petroleum Engineers Journal, 49-55 (1964)

11. J. Colonna, F. Brissaud, and J.L. Millet. "Evolution of capillarity and relative permeability hysteresis". Society of Petroleum Engineers Journal, 12, 28-38 (1972).

12. R. Fulcher, E. Turkay and C. Stahl. "Effect of capillary number and its constituents on two-phase relative permeability curves". SPE 21170-PA (1985)

13. J. Gerhard and B. Keuper. "Relative permeability characteristics necessary for simulating DNAPL infiltration, redistribution and immobilization in saturated porous media". Water Resources Research 39, W1213 (2003)

14. G. Jerauld, and S. Salter, S. J. "The effect of porestructure on hysteresis in relative permeability and capillary pressure". Transport in Porous Media, 5, 103-151 (1990).
15. P. Valvatne and M. Blunt. "Predictive pore-scale modelling of two-phase flow in mixed-wet media". Water Resources Research 40, W07406, (2004).

16. A. Kjosavik, J. Ringen, and S. Skjaeveland. "Relative Permeability Correlation for Mixed-Wet Reservoirs". SPE Journal, 7, 49-58 (2007).

17. L. Andersson, S. Schlueter, and D. Wildenshild. "Defining a novel pore-body to pore-throat 'Morphological Aspect Ratio' that scales with residual non-wetting phase capillary trapping in porous media". Advances in Water Resources, 122, 251-26. (2018)

18. Schembre-McCabe, R. Salazar-Tio, and J. Kamath, 2012. "Two examples of adding value through Digital Rock Technology”. SCA 2012-081 (2012)

19. R. Farokhpoor, E. Westphal, N. Idowu, P. Oren and B. Fletcher. "Gas-water steady-state relative permeability determination with two approaches; experimental and digital rock analysis, strengths and weaknesses". SCA2016-12. (2016)

Table 1. Properties of the three representative sandstones, and their LET function parameters for the gas Rel Perm [7]

\begin{tabular}{|c|c|c|c|}
\hline & Porosity p.u. & $\begin{array}{c}\text { Dry Gas } \\
\text { Perm. } \mathrm{mD}\end{array}$ & L,E,T for $k_{\mathrm{rg}}$ \\
\hline S22B & 20.2 & 107 & $2.2,0.09,1.5$ \\
\hline S26B & 17.1 & 9.4 & $0.8,0.4,2.0$ \\
\hline Berea C & 21.8 & 665 & $0.7,0.67,1.8$ \\
\hline
\end{tabular}

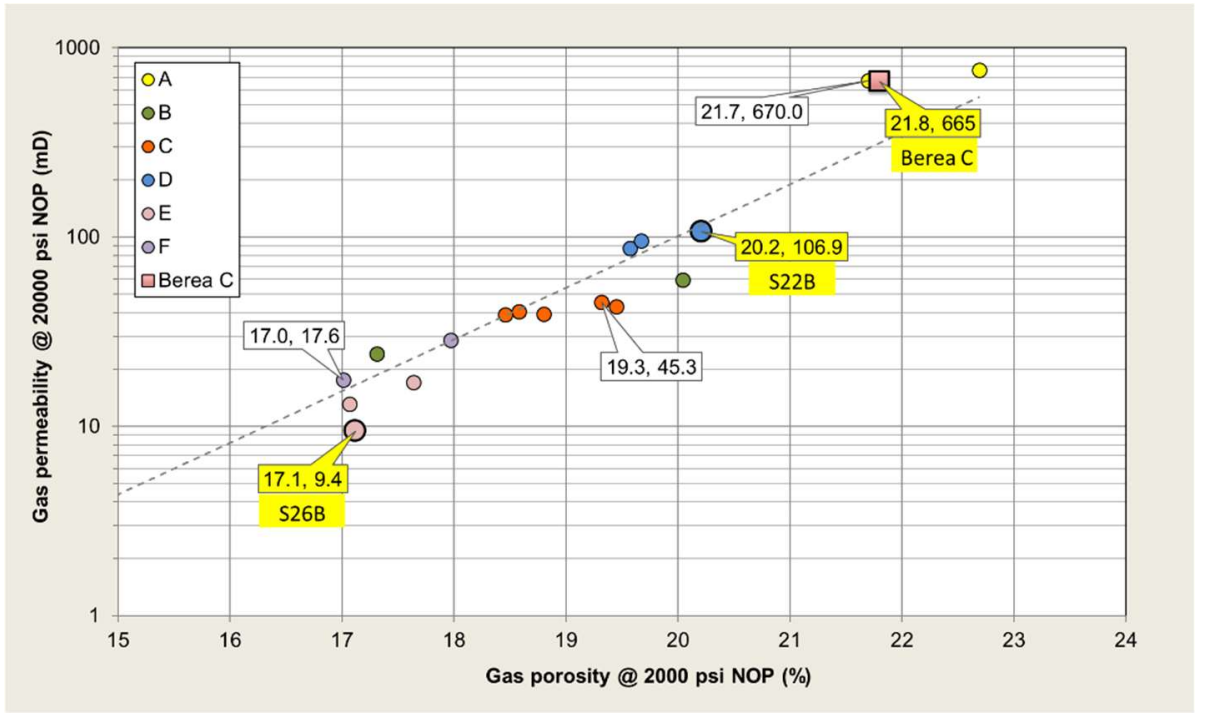

Fig. A1. Dry nitrogen porosity and permeability of the five tested samples from NWS gas field, measured at 2000 psi net overburden pressure. The set of samples represents the range of routine core analysis values of medium to good reservoir quality. The Berea $\mathrm{C}$ sandstone has a porosity and permeability very similar to the highest permeability sample among those tested from the reservoir set. The three samples for which relative permeability results are presented in this paper are highlighted in yellow. 


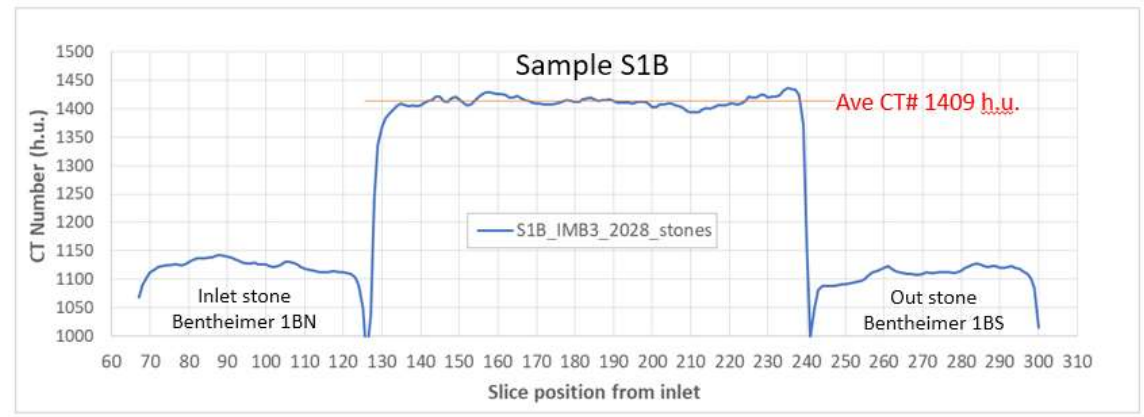

Fig. A2. Profile of average X-ray CT numbers through the assembly of inlet stone-sample-outlet stone mounted in the test rig, prior to commencing measurements. The sample has a uniform brine saturation of $30 \%$ and is in capillary equilibrium with the stones. The dip in CT number at the boundary marks the position of the single ply Kimwipe tissue used to maintain good continuity of the brine phase between the end faces of rocks.

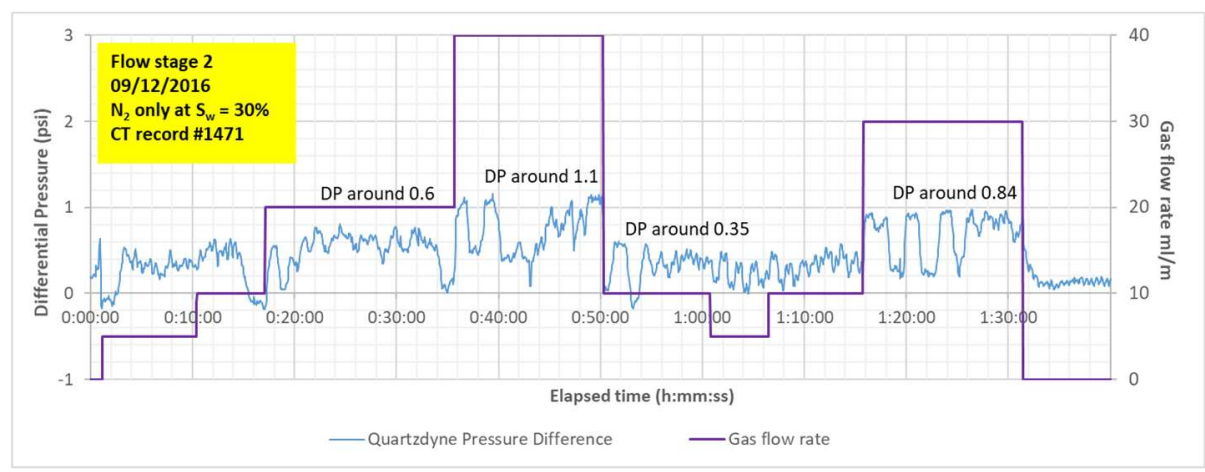

Fig. A3. Example of a completely unstable pressure record during multiple rate gas flow experiments in sample S22B. This problem was rectified by bleeding off any brine droplets that had entered the PEEK tubes connected to the flow transducers.
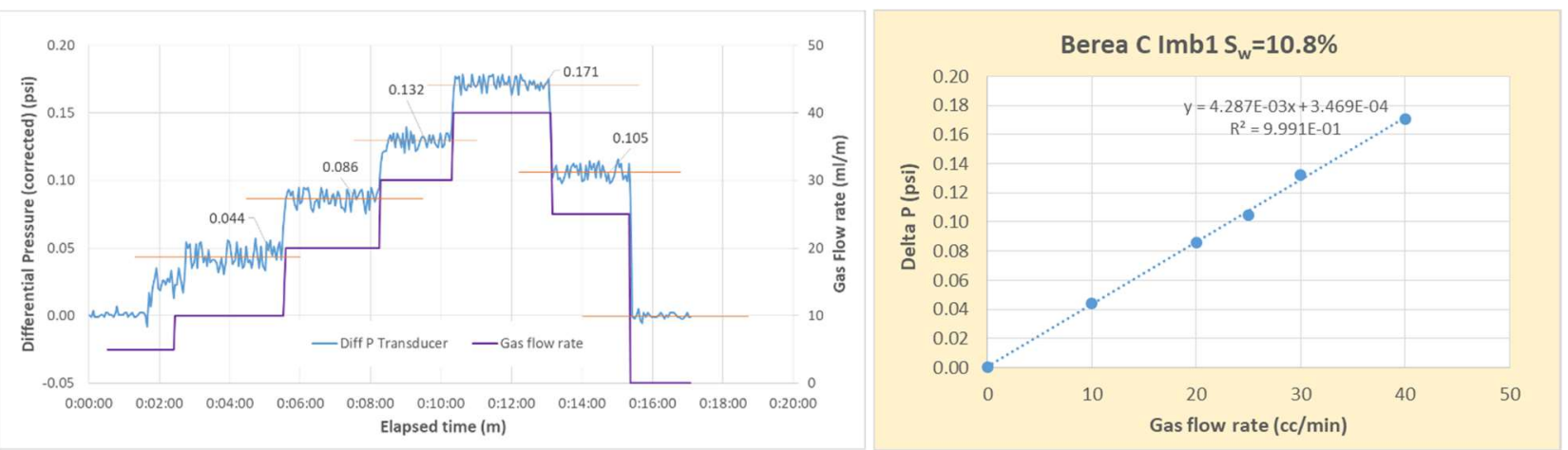

Fig. A4. Example of stable flow-pressure record in sample Berea C, at multiple gas flow rates and a plot showing linearity of the Darcy flow regime. The static periods of no flow show the very high resolution and low noise level of the differential transducer. The fluctuations around the mean pressure values during gas flow are real "flutter" in the multiphase flow process. 


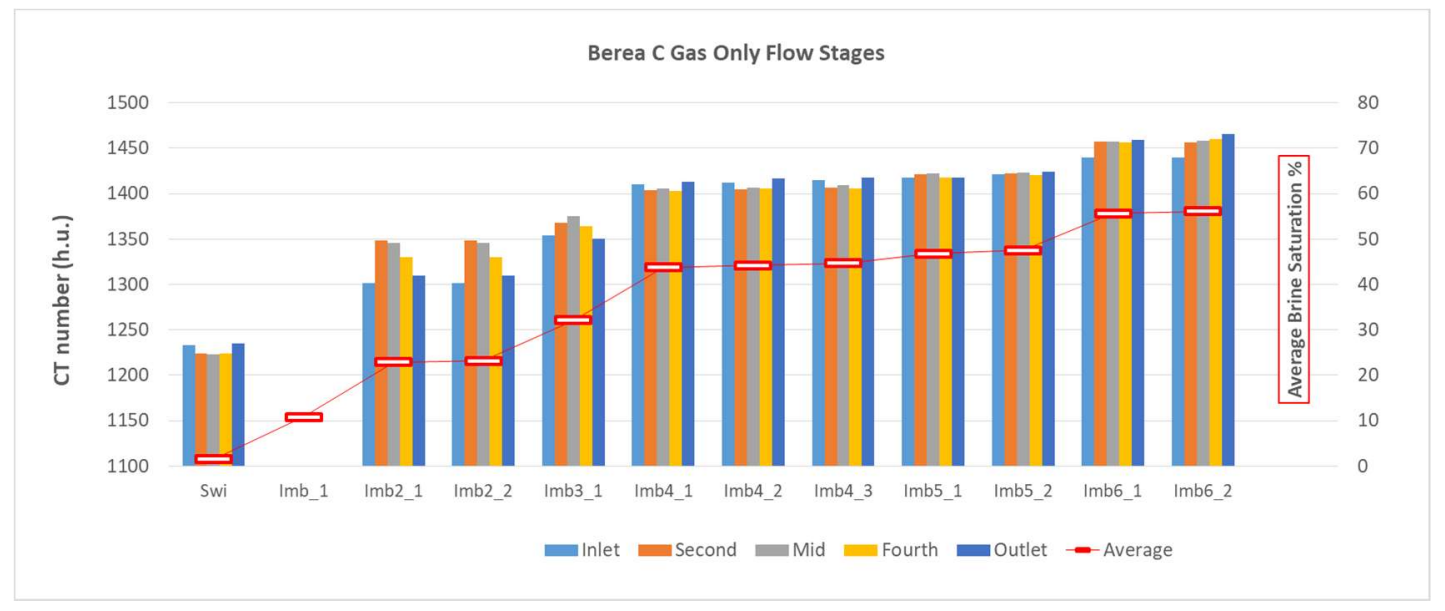

Fig. A5. Saturation distribution through the Berea $\mathrm{C}$ sample during tests with gas-only flow. The average CT values for 5 regions running from inlet through to outlet are shown here for simplification, although the data were collected with a slice thickness of around $0.5 \mathrm{~mm}$, as seen in the following figure. For Imb 1, x-ray CT data was not collected, but the brine distribution was measured before loading into the rig, when it was uniform, and then after testing, blow-down and extraction, when the brine was found to have retracted towards the mid-point of the plug. For the two CT scans in Imb2, brine again was initially uniform as determined by NMR profiling, prior to loading into the rig, but retracted away from the core ends. This is seen very slightly in the third imbibition stage, and thereafter the brine distributions were highly uniform apart from small end effects, as seen in the following figure.

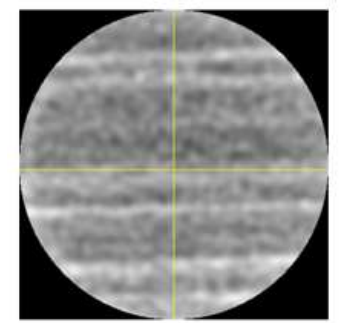

Transverse

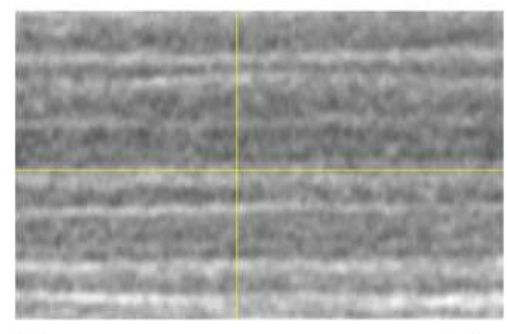

Inlet

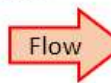

Lateral view

Outlet

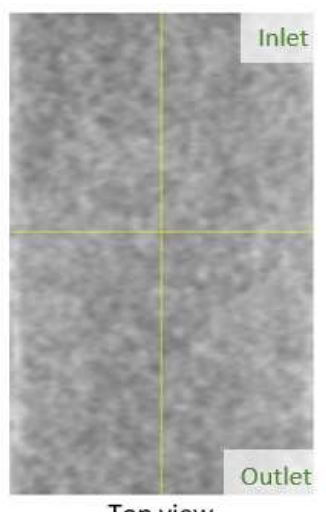

Top view

(a)

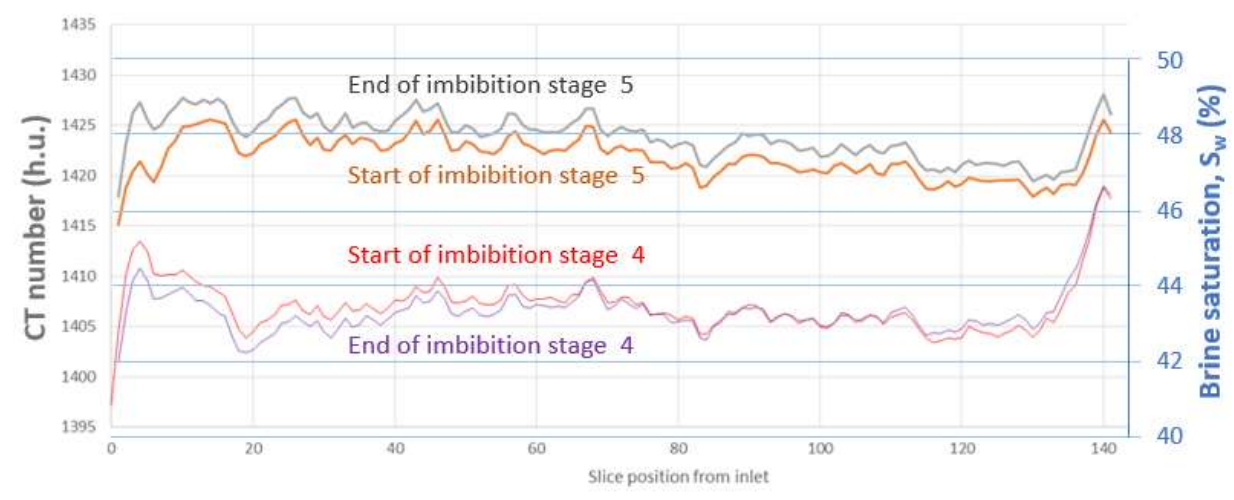

(b)

Fig. A6. (a) X-ray CT images of Berea C Sample during imbibition stage 4 , at around $43 \%$ average brine saturation. The distribution of brine appears to be very uniform. The horizontal laminations are well defined, and the contrast between them is highest at intermediate saturations owing to the finer laminae having a higher brine content than the coarser-grained laminae. (b) Detail of x-ray profiles showing average CT number variations from inlet to outlet in imbibition stage 4 , and the subsequent stage after a further increment of blotting. Small capillary end effects are evident that do not seem to have affected flow or pressure data. While there was essentially no change in brine saturation during stage 4 , there was a small net transfer of brine into the sample from the end stones during stage 5, even during gas-only flow, but this amounted to an overall increase in saturation of less than 1 percent. 


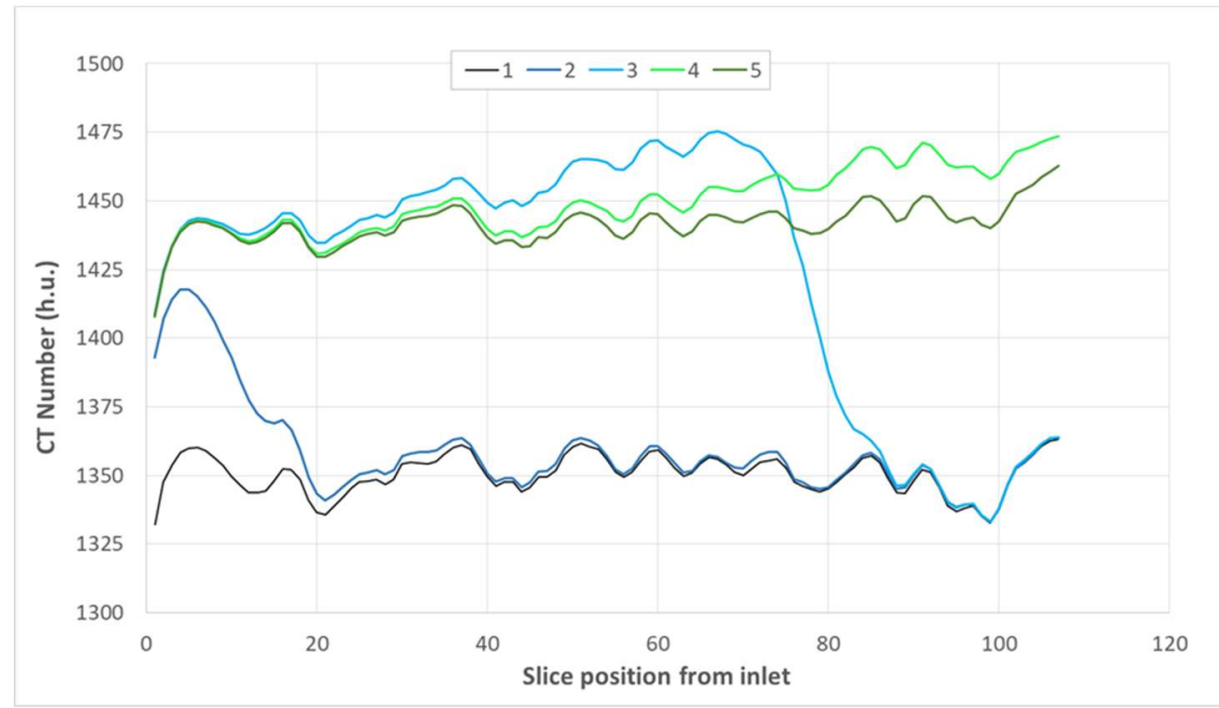

Figure A7. CT value evolution during brine imbibition stages from $\mathrm{S}_{\mathrm{w}}=22 \%$ in sample $\mathrm{S} 22 \mathrm{~B}$, during co-current flow at 1000:1. The initial uniform distribution of brine in Image 1 becomes banked at the inlet (2), and a large front progresses two thirds of the way into the sample (3) before reaching the outlet and dissipating (4) and becoming nearly uniform again by Image 5 . This figure illustrates the development of the significant gap in relative permeability data for situations with an even brine saturation when using co-current flow, even at high gas rate $(60 \mathrm{ml} / \mathrm{min})$ and very low brine fraction.

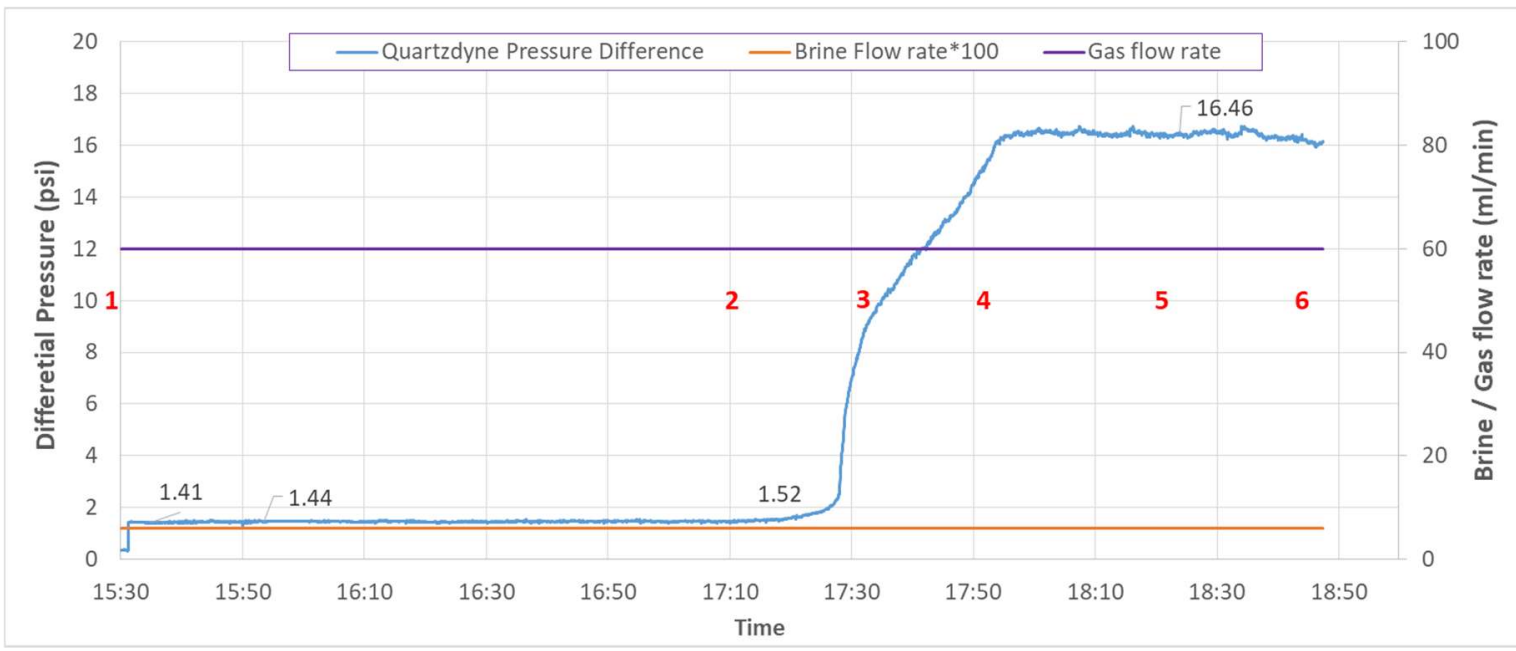

Figure A8. Pressure record for initial gas flow in sample S22B commencing from $\mathrm{S}_{\mathrm{w}}=22 \%$, followed by high gas fraction co-current flow (1000:1 ratio) that matches to the saturation history shown in the previous figure. There are no good Rel Perm data points between an initial gas permeability of $103 \mathrm{mD}$, and the drop right down $2.2 \mathrm{mD}$ after the brine spreads out at an average brine saturation of $49 \%$ (Image 5 in the previous figure, banked point in Figures 5 and 6 ). 


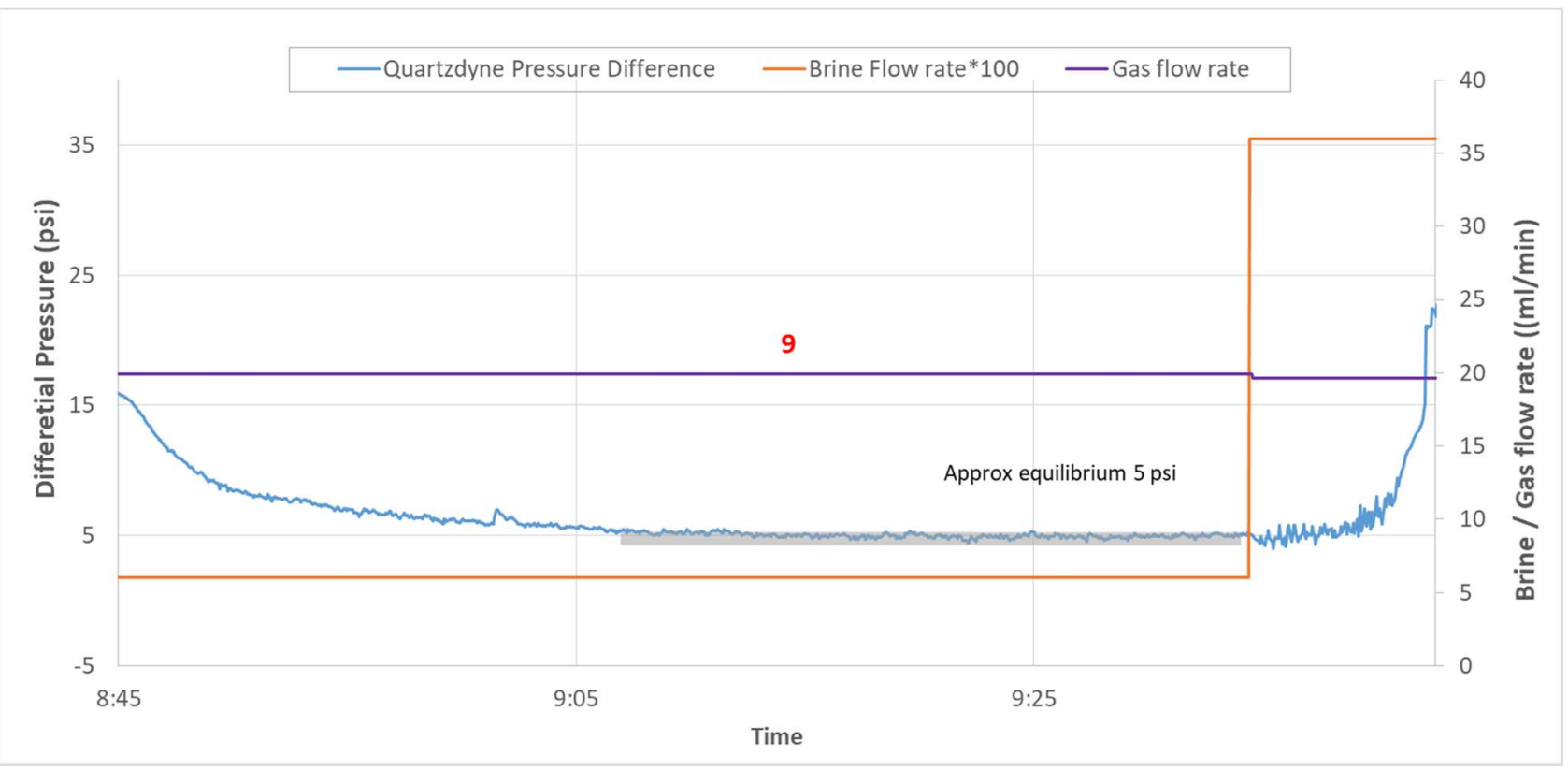

Figure A9 After the sample S22B was left overnight, co-current flow was recommenced at a ratio of 333:1. The permeability to gas recovered to $7.6 \mathrm{mD}$ and flow was very stable. Brine saturation was uniform and had progressed slightly to 53\% (the next valid Rel Perm point in Figures 5 and 6). A relatively small redistribution of the brine in the sample significantly affected its gas permeability, as can be seen from the drop off in differential pressure from the peak of 16.5 psi down to 5 psi during this flow stage. 